\title{
Stream Flow Painting from Real Images Using Anisotropic Band-Pass Filter
}

\author{
Xiaohua Zhang ${ }^{1}$, Ning Xie ${ }^{2}$, Yuelan $\mathrm{Xin}^{3}$, Heming Huang ${ }^{4}$ \\ ${ }^{1}$ Department of Information System and Management, Hiroshima Institute of Technology, Hiroshima, Japan \\ ${ }^{2}$ School of Software Engineering, Tongji University, Shanghai, China \\ ${ }^{3}$ Department of Physics, Qinghai Normal University, Xining, China \\ ${ }^{4}$ School of Computer Science, Qinghai Normal University, Xining, China
}

\section{Email address:}

zhxh@cc.it-hiroshima.ac.jp (Xiaohua Zhang), seanxiening@gmail.com (Ning Xie), xinyue001112@163.com (Yuelan Xin), huanghm@qhnu.edu.cn (Heming Huang)

\section{To cite this article:}

Xiaohua Zhang, Ning Xie, Yuelan Xin, Heming Huang. Stream Flow Painting from Real Images Using Anisotropic Band-Pass Filter. Science Journal of Circuits, Systems and Signal Processing. Special Issue: Computational Intelligence in Digital Image Processing.

Vol. 3, No. 6-1, 2014, pp. 30-38. doi: 10.11648/j.cssp.s.2014030601.15

\begin{abstract}
A new non-photorealistic rendering algorithm is proposed for creating artistic painting with soft stream flow from natural color images. The algorithm consists mainly of two stages, that is, a revised bilateral filter called trilateral filter is firstly applied to original color image for creating drawings using gradient information and then a DoG-like band-pass filter is adapted for generating soft stream flow along the eigenvectors and therefore the image is smoothed along curved stream lines. The proposed trilateral filter is an extension of bilateral filter by incorporating gradient space. On the other hand, DoG-like band-pass filter is designed by applying eigenvectors and eigenvalues of a structure tensor matrix calculated at each pixel. Our approach effectively preserves image main structures while smoothing image regions in an anisotropic way. Even in regions with lower contrast, stream flow-like potential structures are also well produced due to a gradient relaxation. The experiments demonstrate that the proposed algorithm works well and produces good and pleasant visual results.
\end{abstract}

Keywords: Anisotropic Filter, DoG-Like Band-Pass Filter, Trilateral Filter, Stream Flow Painting

\section{Introduction}

Non-Photorealistic Rendering(NPR) is still attracting many researchers to devote themselves to this interesting field [1,2], although it has been studied over many decades when it had come to represent research areas of computer graphics and image processing. Developing new techniques to create artistic drawing or painting from real images in different way is still endless. The aesthetics of artistic painting attributes to its perceptual ambiguity than photographs. Artistic paintings have a characteristic charm beyond photographic in some sense for appreciation.

It is known that there are several hot topics in the field of NPR. In the computer graphics community, many researchers have spent much effort for approximating artistic stippling often used in book illustration to simulate shading or varying degrees of solidity by using small dots [3,4,5]. Some of algorithms draw dots with same or different sizes and different densities according to the shading in the given input image.
Other algorithms arrange dots guided by the image features such as edges or corners. Computer-generated line drawing is another artistic representation style, aiming at simple yet effective visual communications. Many methods of this kind of rendering are reported recently, such as interactive pen-and-ink illustration [6], suggestive contours [7], apparent ridges [8], coherent line drawing [9] and abstract line drawing [10]. Other technique is for paying more attention to stroke-based rendering (SBR) [11] to simulate hand-painting images. Some of excellent researches are, for example, painterly rendering [12] and image parsing [13]. Recently automatic hand-painting generation has been deeply studied using image filters by setting several necessary parameters. Many filtering methods are reported, such as artistic edge and corner enhancing [14], flow-based abstraction [15], and shock filter depended enhancing approach [16].

In this research work, we also developed an approach for creating artistic painting with soft stream flow by applying image filtering method. Our filter consists of two components which are applied to the input color image in two stages 
sequentially. In the first stage a trilateral filter is applied to original image. A trilateral filter is an extension of the traditional bilateral filter. The trilateral filter measures weights not only in image geometrical domain space and color range space but also in gradient space. The weight in the gradient space for each pixel ensures that a drawing can also be proceeded in the direction vertical to the edge orientation to simulate a wide brush. In the second stage a band-pass anisotropic filter is applied to the result image created by the first stage. Even lower contrast regions can be smoothed by the band-pass anisotropic filter. This filter not only preserves edges but also enhances edges. The band-pass anisotropic filter is similar to the Difference of Gaussian (DoG) [17], but it is an anisotropic one applied in an elliptical region for each pixel in image domain space. The band-pass filter was designed depending on the eigenvalues and eigenvectors of a structure tensor matrix calculated at each pixel. The useful information about image feature such as obvious edges and corners were used during filtering. Due to the trilateral filter is firstly applied to the image, the band-pass filter creates more abundant stream flow like painting effects. Moreover, the stream flow is in a soft way unlike shock filter, which produce hard, shock stream flow.

\section{Related Work}

It is known that artistic painting automatically from real images by filtering means had been researched for long time. The most famous filter is probably the bilateral filter [18] which is closely related to our technique, although its original goal is to smooth image while preserving edge and corner features. The popular bilateral filter over image geometrical domain space and color range space has been adapted for many applications such as photograph enhancement and detailed texture removal. Recently an explicit image filter called guided filter was proposed [19]. The guided acts as an edge-preserving operator like bilateral filter, but it behaves better near edges and fast. Kuwahara filter [20] in a squared neighborhood was modified to decrease the limitations of the unstable subregion selection by using sectors of disc and achieved good artistic edge and corner enhancing [14]. Moreover, by defining weighting function over sectors of disc and over sectors of ellipse, anisotropic Kuwahara filter was proposed for image and video abstraction [21]. Structure adaptive filter for image abstraction is a different method which filters image in a fast separated way over an elliptic region along gradient direction and then along edge tangent direction [25].

Kass reported a non-photorealistic rendering technique making use of random variation. The coherent noise was computed using a specialized filter [22]. Zhao proposed an interactive control of perceptual entropy for abstract painting from real images [23]. Painterly rendering for Sumi-e is also reported [24, 25] which simulates artist's actions by using reinforcement learning to generate strokes.

It is also reported that many researches are about using diffuse or shock filter [26] to produce simplified artistic illustration from color images [27,28]. However, the shock filter seems to generate noticeable typical high contrast at the shock edges, the stream flow is very hard and shock and looks so stiff and inflexible. More pleasant and harmonic results were achieved with gradient-directed shock filter [16, 29]. The result images are with more balanced color contrast and consistent diffuse color variation. Reference [30] also introduced a method simplifies both shapes and colors simultaneously. The method is based on mean curvature flow and combining with shock filter.

Trilateral filter is not a new concept, which is an extension of bilateral filter. It is proposed [33] to design a single-pass nonlinear trilateral filter for high contrast images and meshes, which aimed at edge-preserving smoothing and visual detail removal for multiple dimensional signals. In addition to considering weights in image geometrical domain space and range space just like bilateral filter, this trilateral one filters gradient space of input image to find a local plane of intensity and then computes adaptive neighborhood for trilateral filtering local detail signals and adds them to the original signal. Later, a fast approximation of trilateral filter for tone mapping was reported [34], which is about 15 to 25 times faster than the original trilateral filter [33]. The tone mapping for high dynamic range image is established based on fast approximation of bilateral filter for computing weights in gradient space and the down sampling algorithm for computing tilting angle image.

Another trilateral filter was reported for biomedical images [35], the filtering process was with a few iterations. This filter is also an extension of the famous bilateral filter by considering local structure similarities and it can smooth image within a narrow window while preserving edges.

Although these trilateral filters in references using different third weight functions applied to local detail signals, their purposes are to smooth images while preserving edges. These filters add the filtered detail signal to the original signals [33, 34] or using local structural information as the third weighting function applied to the image iteratively [35]. Aiming at creating stream flow painting, we design a different trilateral filter and apply it to the input image directly and then an anisotropic band-pass filter is employed to generate the potential soft stream flow even in low contrast region to enhance the artistic effect.

\section{A Brief Overview}

Our goal is to design an image filter which automatically creates artistic painting with soft stream flow from natural color image, given a few of controllable parameters. We extends bilateral filter to construct a trilateral filter and apply it to natural color input image by establishing weighting functions not only over image geometrical space and color range space but also over image gradient space. In some image regions with almost uniform color or intensity, the gradient magnitudes are near to zero. That means the gradient orientation is unstable and is not suitable for guiding painting direction. Even in this case, our algorithm can produce the 
potential stream flows using information from the surrounding of regions with uniform colors. We use an iterative gradient relaxation method to compute gradients with smaller magnitude by considering their neighborhood. This relaxation is similar to the technique used in image inpainting [31] for removing or replacing unwanted object and repairing damaged photographs or images. In the uniform regions with low contrast, since feature information is not enough to

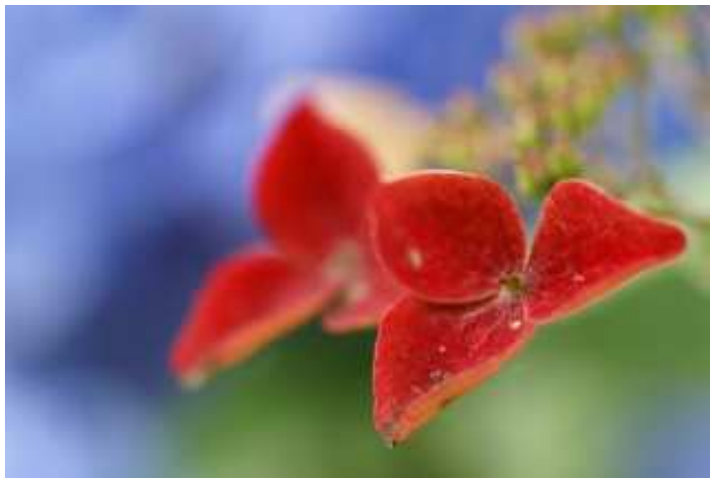

(a)

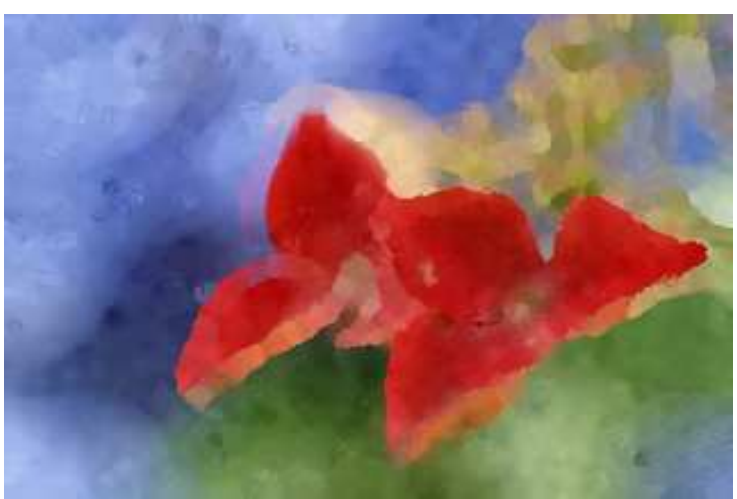

(c)

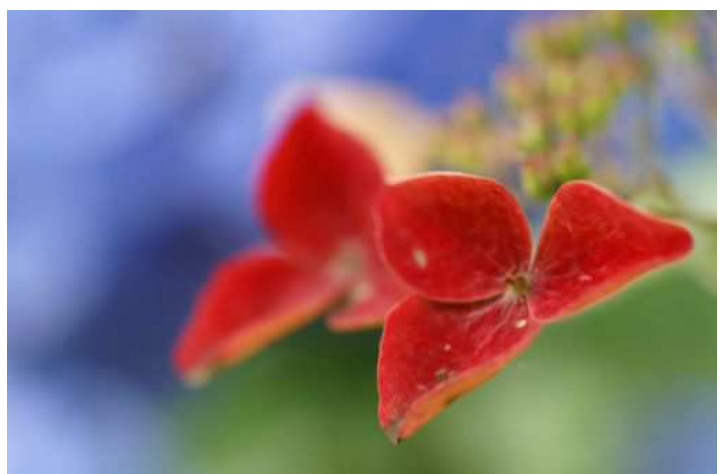

(e) generate painting effect, we add Gaussian noise to disturb colors. This can be considered as an addition of ink to the canvas. As an example, the result of trilateral filtering at the first stage is illustrated in Fig.1 (c). The original image is shown in Fig. 1(a). By observation, one can know that most of region in the original image is low contrast and with almost uniform color.

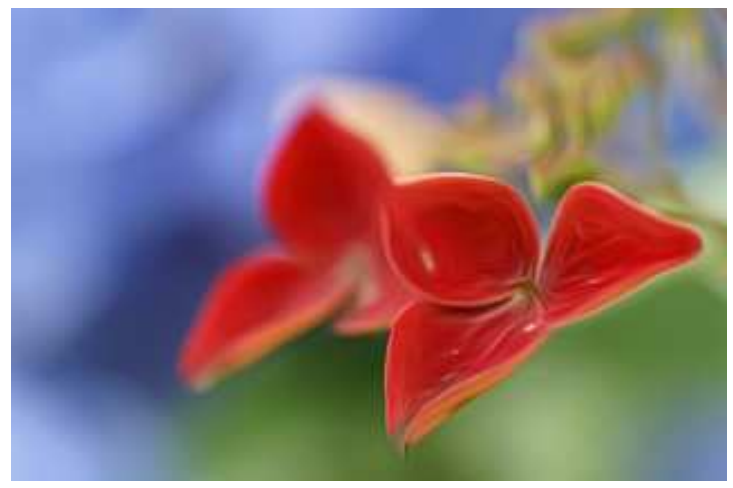

(b)

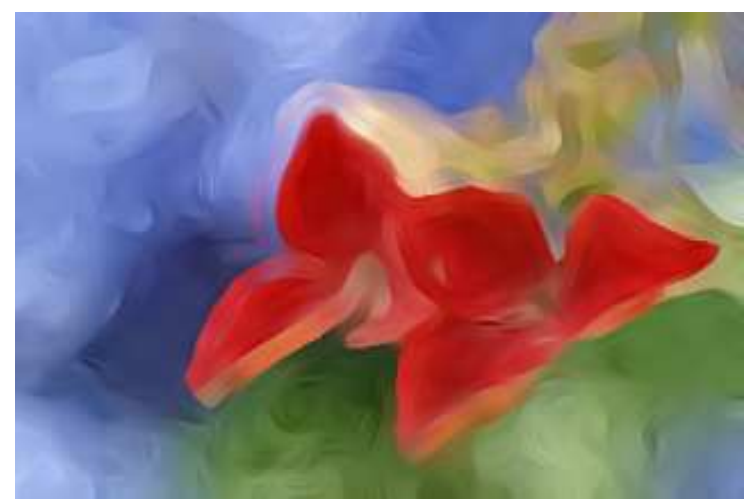

(d)

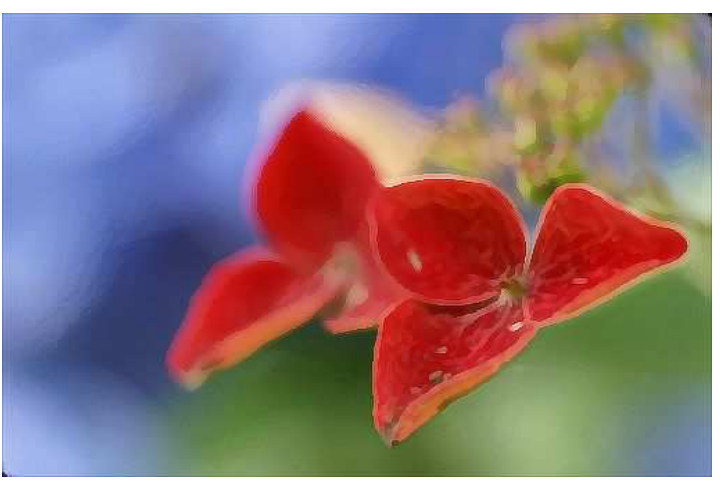

(f)

Figure 1. Filtered results at the different step: (a) an original image named the Three Primary Colors; (b) anisotropic band-pass filtered result. Note that it is too clean and it is hard to say it a painting like image; (c) the intermediate result after the first step finished, some drawings are generated in the low contrast regions; (d) anisotropic band-pass filtered result in the final step. It contains soft stream flows and is more like an artistic painting than (b). For comparisons, (e) is a result created with anisotropic bilateral filter and $(f)$ is a result with stiff stroke produced by shock filter.

The next stage in the algorithm is to apply an anisotropic band-pass filter to the result obtained at the first stage. The band-pass filter is defined over an elliptical region calculated from tensor matrix at each pixel. Tensor space is computed from the relaxed gradient space. The tensors are also relaxed in a similar way as in the first stage. The purpose of relaxation is to avoid the case of nearly zero tensor having zero eigenvalues and zero eigenvectors which cannot direct 
painting to a correct orientation. The anisotropic band-pass filter is very similar to the bilateral filter, but the weight function in image space is analogy to Difference of Gaussian (DoG) function. However, the two Gaussian functions are computed with eigenvectors and modified eigenvalues. Fig. 1(d) shows a final result by applying the anisotropic band-pass filtered to Fig. 1(c). It is observed that soft stream flows were produced in most of low contrast region although they are almost with uniform color where one cannot observe stream flow in the original image. Fig. 1(b) is a filtered result using anisotropic band-pass filter without weighting in gradient space, that is, an anisotropic band-pass bilateral filter. By comparing Fig. 1(d) with Fig. 1(b), one can observe the differences between the two filters.

\section{Trilateral Filter}

Bilateral filter works well for smoothing image while preserving edges. However, it cannot produce abundant context in low contrast regions. Gradient information is very useful for producing stream flow. The proposed trilateral filter extends bilateral filter by considering the weighting function in gradient space, and intends to try to paint along the orientation vertical to edges to disturb uniform region proportionally to the magnitude of gradient to create artistic effect. The filter described in this paper works along the same line as the famous bilateral filter. Since we employ three different weighting functions, we call this filter as trilateral filter, although it is in a different manner with [33, 34, 35] and with different purpose.

\subsection{Bilateral Filter}

Bilateral filter can smooth image while preserving the edge and corner features in a weighted average way. The weighting functions are calculated in both image geometrical domain space and color range space. Larger weights are given to pixels that are closer in image space and pixels whose values are similar in range space. Suppose the given image is denoted as $I(\vec{x})$, where $\vec{x}=(x, y)$ represents position of a pixel, while $I$ represents a scalar intensity at $\vec{x}$ for gray scale image or a vector $I(\vec{x})=\left(I_{r}(\vec{x}), I_{g}(\vec{x}), I_{b}(\vec{x})\right)$ for color image. Suppose the output at this pixel is $I^{\prime}(\vec{x})$, bilateral filter can be written as

$$
I^{\prime}(\vec{x})=\frac{\sum_{\Omega} w_{e}(\vec{x}, \vec{y}) w_{c}(I(\vec{x}), I(\vec{y})) I(\vec{y})}{\sum_{\Omega} w_{e}(\vec{x}, \vec{y}) w_{c}(I(\vec{x}), I(\vec{y}))} .
$$

The parameters and items in expression (1) are defined as follows. $\Omega$ represents a neighborhood region centered at $\vec{x}$, and $\vec{y}$ represents location of pixel in the neighborhood region. $w_{e}(\vec{x}, \vec{y})$ is a Gaussian weighting function with standard deviation $\sigma_{e}$ in image geometrical domain space, while $w_{c}(I(\vec{x}), I(\vec{y}))$ denotes a Gaussian weighting function with standard deviation $\sigma_{c}$ in color range space, which suppresses the contributions from pixels with larger difference to the center pixel. For the filtering, both parameters are very important, since $\sigma_{e}$ controls the smoothing and $\sigma_{c}$ control the preservation of features such as edges and corners. It is known that bilateral filter will degenerate into general Gaussian filter when applied to low contrast regions with almost uniform colors.

\subsection{Trilateral Filter}

Unlike the existed approaches mentioned above, our trilateral filter is constructed by including weighting function over a neighborhood region in a gradient space. It is expected that the painting is also proceeded along an orientation vertical to the edge. This will create some blocky effect, especially on the low contrast regions with almost uniform colors. Suppose the gradient space be computed as $g(\vec{x})=\nabla I(\vec{x})$. Inspired by [16], instead of using the popular Sobel filter, the following rotational symmetric derivative filter kernels are used for approximating the gradient at each pixel:

$$
K_{x}=0.5 *\left(\begin{array}{ccc}
p & 0 & -p \\
1-2 p & 0 & 2 p-1 \\
p & 0 & -p
\end{array}\right), K_{y}=K_{x}^{T},
$$

where $p=0.1875$. The gradients are then computed by convoluting in $x$ and $y$ directions individually as $I_{x}=K_{x} * I$ and $I_{y}=K_{y} * I$.

After computing gradients $g(\vec{x})=g(x, y)=\left(I_{x}(\vec{x}), I_{y}(\vec{y})\right)$, the bilateral filter is easily extended to the trilateral filter:

$$
I^{\prime}(\vec{x})=\frac{\sum_{\Omega} w_{e}(\vec{x}, \vec{y}) w_{g}(g(\vec{x}), g(\vec{y})) w_{c}(I(\vec{x}), I(\vec{y})) I(\vec{y})}{\sum_{\Omega} w_{e}(\vec{x}, \vec{y}) w_{g}(g(\vec{x}), g(\vec{y})) w_{c}(I(\vec{x}), I(\vec{y}))},
$$

The weighting function $w_{g}(g(\vec{x}), g(\vec{y}))$ is defined in the gradient space and also is a Gaussian function as shown in Equation (4) which is similar to the weighting function $w_{e}(\vec{x}, \vec{y})$ in image geometrical space and weighting function $w_{c}(I(\vec{x}), I(\vec{y}))$ in range space:

$$
w_{g}(g(\vec{x}), g(\vec{y}))=\exp \left(-\frac{(g(\vec{x}) \cdot g(\vec{y}))^{2}}{2 \sigma_{g}^{2}}\right) .
$$

Here $\sigma_{g}$ is a standard deviation in the gradient space for computing weighting function, while $(g(\vec{x}) \cdot g(\vec{y}))$ is an inner product between two gradient vectors at two pixels $\vec{x}$ and $\vec{y}$. It is known that if the angle spanned by the two vectors is about $\pm 90^{\circ}$, the inner product is smaller, then the weight become larger, which means that pixels with gradient perpendicular to the gradient of the center pixel have larger contributions to the weighting. An example is shown in Fig. 1(c), smaller blocky regions are created near strong edges. This intermediate result looks like a watercolor painting with lots of irregular blocks with sharper edges. These edges will provide important feature information to the anisotropic band-pass filter in the 
next stage to produce soft stream flow.

\subsection{Gradient Relaxation}

During the above trilateral filtering, it is observed that the magnitudes of gradients are near to zero for some pixels in regions with almost uniform color in the original image. The computed gradients at these pixels are unstable, and may direct painting brush into random orientations. To solve this problem, we adopt a gradient relaxation method to estimate gradients which originally have too small magnitudes. If the magnitude of a gradient is less than a threshold $\phi$, the gradient

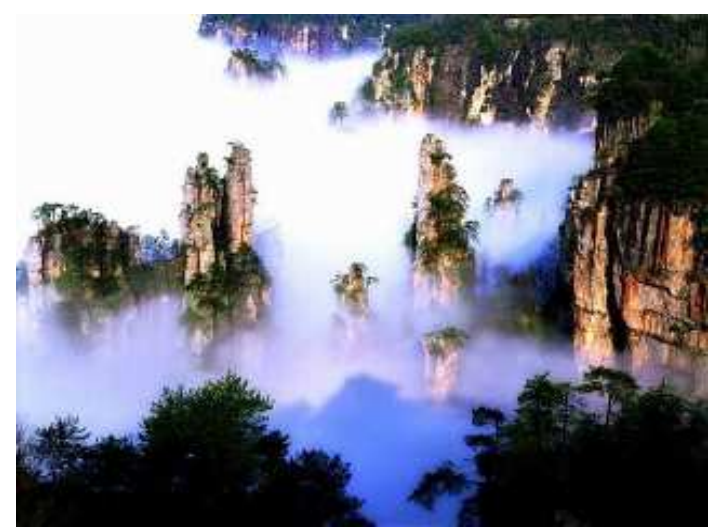

(a)

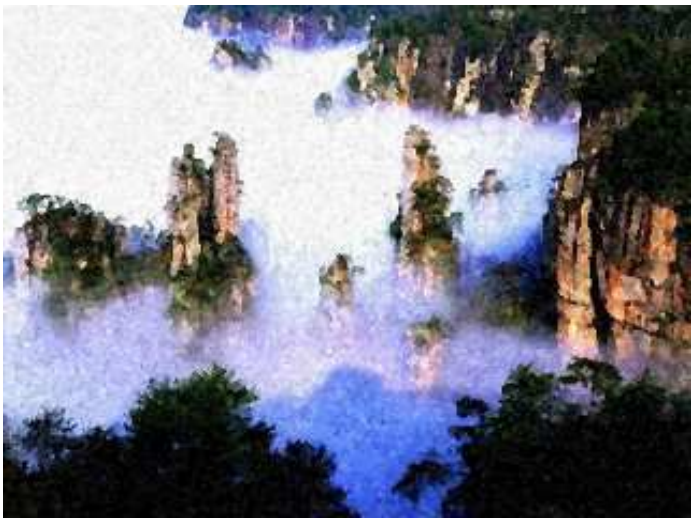

(c)

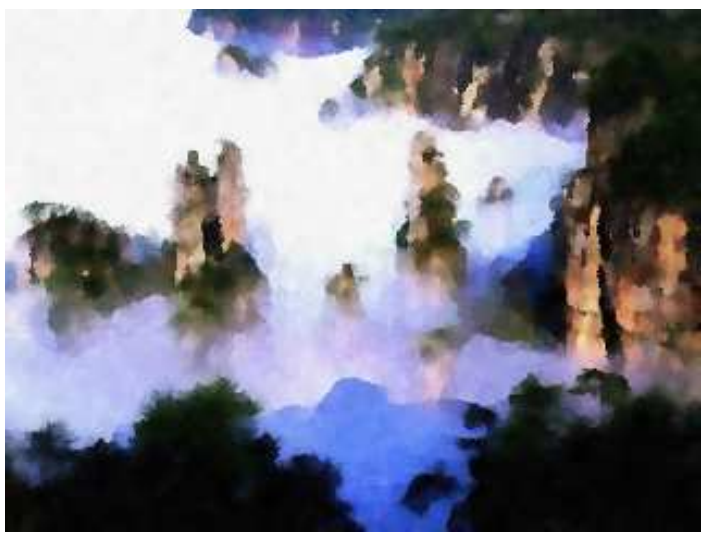

(e) will be computed with relaxation. The computation is an iterative way using following equation:

$$
g_{x, y}^{k+1}= \begin{cases}g_{x, y}^{k} & \text { if }\left|g_{x, y}^{k}\right| \geq \phi \\ \frac{g_{x+1, y}^{k}+g_{x-1, y}^{k}+g_{x, y+1}^{k}+g_{x, y-1}^{k}}{4} & \text { otherwise }\end{cases}
$$

The threshold $\phi$ is empirically set as 0.002 for all experiments in this paper, and $k$ indicates an iterative step

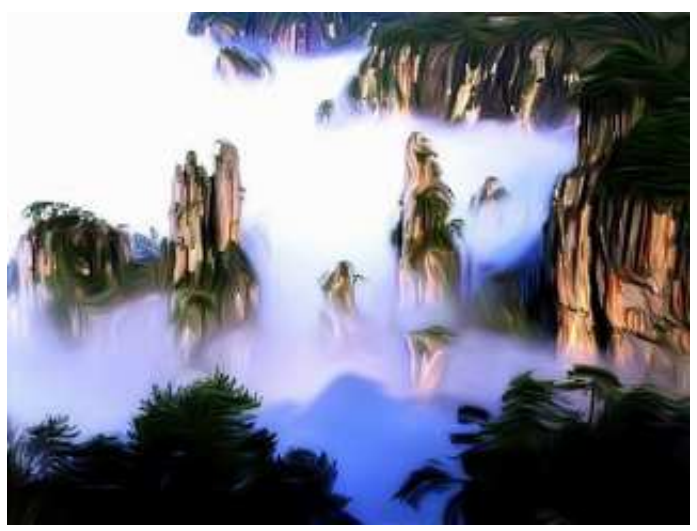

(b)

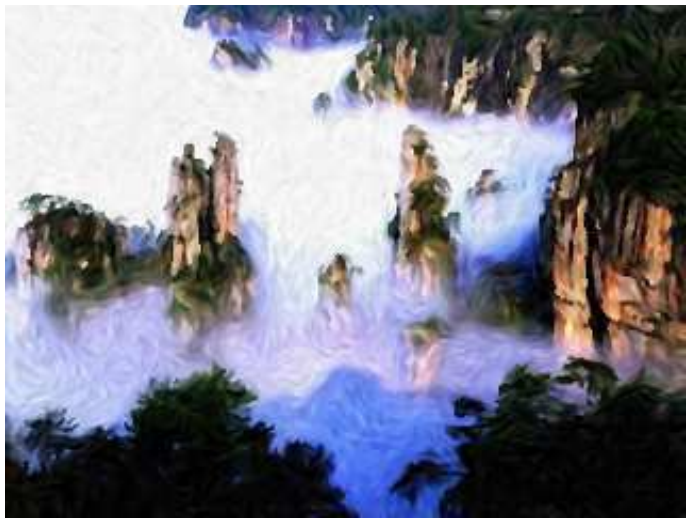

(d)

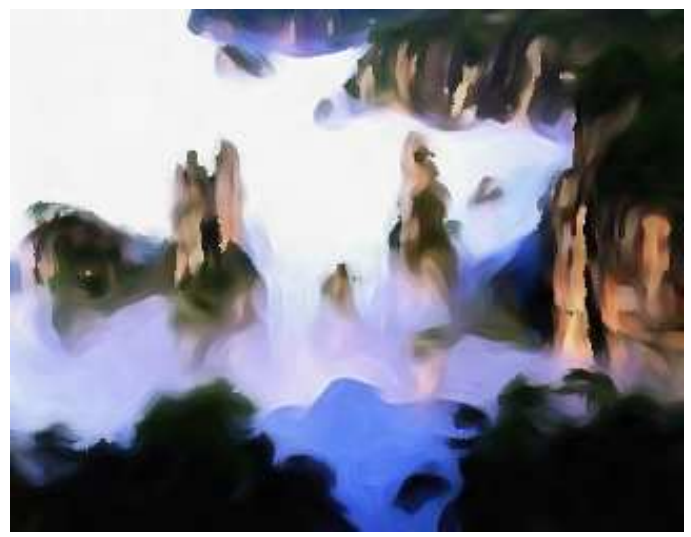

(f)

Figure 2. Experimental results: (a) An original image; (b) Anisotropic band-pass filtered image; (c) Trilateral filter adapted with $\sigma_{g}=0.5$; (d) Anisotropic band-pass filtered result from (c); (e) Trilateral filter adapted with $\sigma_{g}=1.5$; (f) Anisotropic band-pass filtered result from (e). 
with $g_{x, y}^{0}=g(x, y)$ as an initial gradient field. If the magnitude of a gradient is less than $\phi$, it will not be added to the above computation. Note that this relaxation method is only a simple way, although most of gradient vectors can be interpolated, few of them is hard to be computed. We terminate the relaxation when the computation arrives a stable state, which means that no more gradients can be interpolated.

To enhance the artistic painting effect, after relaxing the gradient field, Gaussian noises are added to the original image. The addition of noises can be considered as adding amounts of random pigments or colorants to the canvas to provide abundant textures. This is especially important for low contrast regions with almost uniform colors where lacks textures such as the background in the original image shown in Fig. 1(a). Now we have relaxed gradient field and image with noise added.

\section{Anisotropic Band-Pass Filter}

For creating soft stream flow, we design an anisotropic band-pass filter and apply it to the output from the first stage. A band-pass filter passes frequencies within a certain range and attenuates frequencies outside that range. We use a module similar to the Difference of Gaussian (DoG) [17] to enhance edges, but the filter is defined over an elliptical region. Inspired by the research work in [32], the anisotropic property is obtained by redefining the weighting function in the bilateral filter or trilateral filter as follow:

$$
w_{e}(\vec{x}, \vec{y})=\exp \left(-\frac{(\vec{x}-\vec{y}) D(\vec{x})(\vec{x}-\vec{y})^{T}}{2 \sigma_{e}^{2}}\right) .
$$

Where $D(\vec{x})$ is a semi-definite symmetric matrix and is defined in an elliptical region centered at pixel $\vec{x}$ in image geometrical space. The smoothing is proceeded mainly along the long axis of ellipse. Matrix $D(\vec{x})$ is calculated from eigenvectors and modified eigenvalues of tensor at pixel $\vec{x}$. It is noted that when all matrices at every pixel become unit matrices, the proposed trilateral filter degenerate into the original bilateral filter.

\subsection{Structure Tensor}

To compute matrix $D(\vec{x})$ in equation (5), a tensor space is first calculated. A tensor at each pixel $\vec{x}$ can be found as $T(\vec{x})=g(\vec{x}) g^{T}(\vec{x})$, that is,

$$
T(\vec{x})=\left(\begin{array}{cc}
I_{x}^{2} & I_{x} I_{y} \\
I_{x} I_{y} & I_{y}^{2}
\end{array}\right)
$$

The symmetrical tensor owns a larger eigenvalue $\sqrt{I_{x}^{2}+I_{y}^{2}}$ and the smaller eigenvalue is always 0 . Practically, the above structure tensor at each pixel is smoothed by using Gaussian function with a standard deviation $\rho$ and the smoothed tensor is rewritten as follow:

$$
T_{\rho}(\vec{x})=K_{\rho} *\left(\begin{array}{cc}
I_{x}^{2} & I_{x} I_{y} \\
I_{x} I_{y} & I_{y}^{2}
\end{array}\right)=\left(\begin{array}{cc}
A & B \\
B & C
\end{array}\right) .
$$

Where $K_{\rho}$ is a Gaussian kernel with a standard deviation $\rho$. Similar to gradient relaxation, the structure tensors with smaller magnitudes are also relaxed for stable computation. This is because in some regions with lower contrast, that is, with almost uniform colors, the tensor cannot describe the local region structure accurately.

Since the above tensor matrix is a simple $2 \times 2$ symmetrical one, the eigenvectors $u, v$ and eigenvalues $\mu_{1}, \mu_{2} \quad\left(\mu_{1} \geq \mu_{2}\right)$ can be calculated in an analytical way. $u$ is the major eigenvector corresponding to the eigenvalue $\mu_{1}$, and $v$ is the minor eigenvector corresponding to the eigenvalue $\mu_{2}$. The eigenvalues are

$$
\mu_{1,2}=\frac{A+C \pm \sqrt{(A-C)^{2}+4 B^{2}}}{2} .
$$

Therefore the corresponding eigenvectors are simply computed as $u=\left(B, \mu_{1}-A\right)^{T}$ and $v=\left(\mu_{1}-A,-B\right)^{T}$.

\subsection{Matrix Computation for Weighting Function}

Before computing matrix $D(\vec{x})$ in the weighting function, the eigenvectors of structure tensor are normalized to unit vectors which direct smoothing orientation. As analyzed in [25], the eigenvalues provide very important information about image structures. If pixels are located in regions with low contrast, the eigenvalues are characterized by $\mu_{1}=\mu_{2}=0$, if pixels are on straight edges, the eigenvalues have property $\mu_{1} \gg \mu_{2}=0$, and if pixels are on corners, the corresponding structure tensor provides eigenvalues $\mu_{1} \geq \mu_{2} \gg>0$.

It is noted that the symmetrical tensor matrix can be represents by its eigenvalues and its eigenvectors mathematically:

$$
T_{\rho}(\vec{x})=\mu_{1} u u^{T}+\mu_{2} v v^{T} .
$$

Since the structure tensor contains much of information about the image structure such as edges and corners, the matrix for weighting function is reconstructed similar to the tensor matrix with the same form but with different coefficients:

$$
D(\vec{x})=\lambda_{1} u u^{T}+\lambda_{2} v v^{T} .
$$

We compute $\lambda_{1}$ and $\lambda_{2}$ similar to [23]. However, we do not hope too many stream lines are generated in the low contrast areas, so the eigenvalues of matrix $D(\vec{x})$ are both restricted to the range $(0,1)$ as simply defined by

$$
\lambda_{1}=\frac{\mu_{1}}{\mu_{1}+\mu_{2}}, \quad \lambda_{2}=\frac{\mu_{2}}{\mu_{1}+\mu_{2}}
$$


And $\lambda_{1}=\lambda_{2}=\gamma(\gamma \in(0,1))$ if pixels are in a low contrast region where the tensor is almost zero.

\subsection{Construction of Band-Pass Filter}

Although the above described filter works in an elliptical region in an anisotropic way, like Gaussian filter and bilateral filter, it is substantively a low-pass filter, since it mainly smooth image along edge orientation. We use a method analogy to the one proposed in [32] to construct an anisotropic band-pass filter to smooth image while preserving and enhancing edges. The idea is to decompose the weighting function of equation (5) into two components in a multiplication form as $w_{e}(\vec{x}, \vec{y})=w_{e}^{1}(\vec{x}, \vec{y}) w_{e}^{2}(\vec{x}, \vec{y})$. Note that each of weighting functions are Gaussian function, as an example, the first function is rewritten as

$$
w_{e}^{1}(\vec{x}, \vec{y})=\exp \left(-\frac{(\vec{x}-\vec{y}) \lambda_{1} u u^{T}(\vec{x}-\vec{y})^{T}}{2 \sigma_{e}^{2}}\right) .
$$

The second component can be represented in the same form but using the eigenvalue $\lambda_{2}$ and eigenvector $v$. This second weighting function is responsible for smoothing along edge direction, that is, the minor eigenvector $v$. At the same time, the first weighting function is responsible for smoothing along eigenvector $u$, that is, in the orientation perpendicular to the edge. However, since $\lambda_{1}$ is larger in general compared with $\lambda_{2}$, the weighting function $w_{e}^{1}(\vec{x}, \vec{y})$ decreases very quickly at the distance from pixel $\vec{x}$. A Gaussian of Different (DoG) is constructed to replace this weighting function to detect and then enhance the edges. The exponent part in equation (12) is denoted as $p(\vec{x}, \vec{y})$, then $w_{e}^{1}(\vec{x}, \vec{y})$ is rewritten as

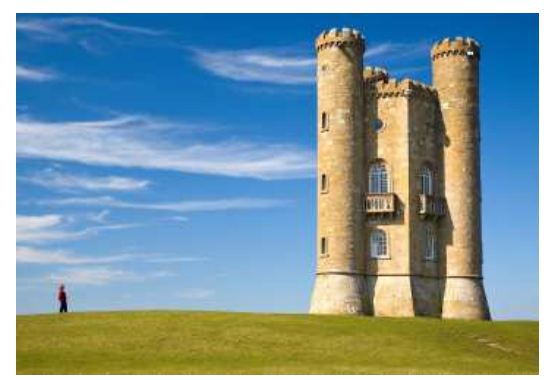

(a)

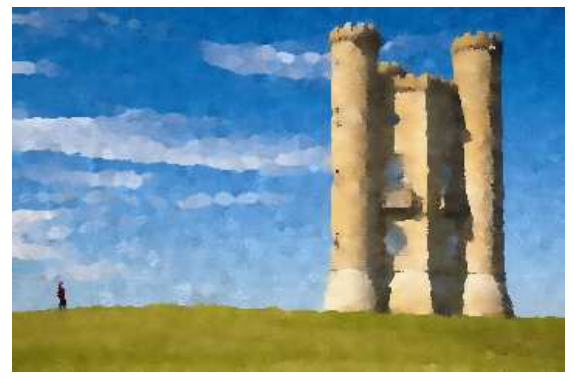

(b)

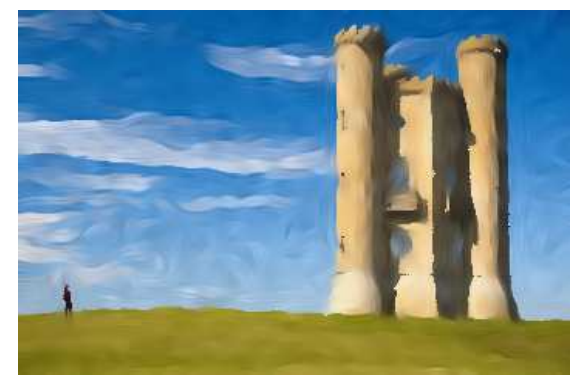

(c)

Figure 3. Experimental results: (a) An original image with a large scale low contrast region; (b) Trilateral filtered result with abundant texture in the low contrast region; (c) Anisotropic band-pass trilateral filtered result, the brush painting is generated and edges are enhanced.

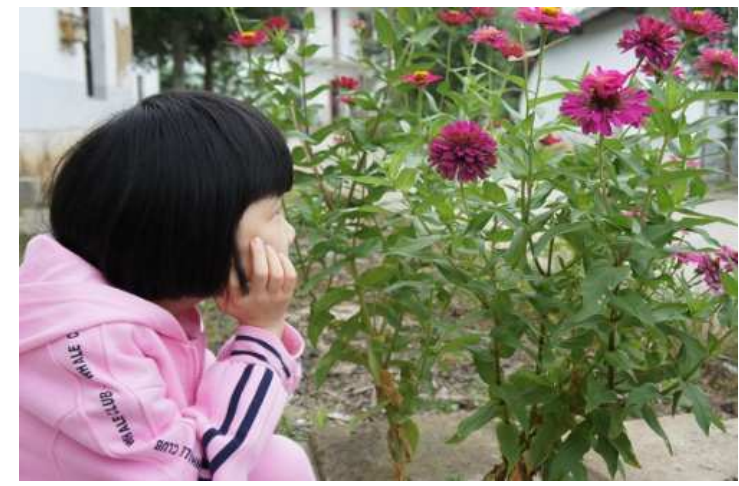

(a)

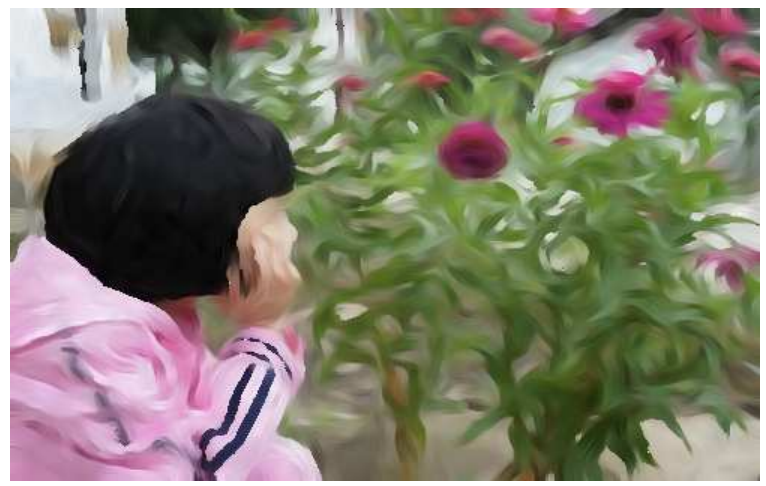

(b)

Figure 4. Experimental results: (a) An original image with a little girl and flowers; (b) Final filtered image.

$$
w_{e}^{1}(\vec{x}, \vec{y})=\zeta_{1} \exp (-p(\vec{x}, \vec{y}))-\zeta_{2} \exp (-0.5 p(\vec{x}, \vec{y})) .
$$

Generally $\zeta_{1}$ is set to be 1.6 in our experiments empirically and $\zeta_{2}$ is computed as $\exp \left(-1.0 /\left(\mu_{1}-\mu_{2}\right)^{2}\right)$ or as 0 if $\mu_{1}=\mu_{2}$. The above filter is expected to have properties of enhancing edges while smoothing low contrast regions along edge directions.

\section{Experimental Results}

To verify the effect of the proposed method, we have implemented the algorithm using the general $\mathrm{C}$ language. The implementation did not use any special feature of graphics. We just designated some needed parameters and run the algorithm automatically.

We have experimented on several images to demonstrate the artistic painting effects, the soft stream flows were well produced. One of results is shown in Fig. 1, several of which were already explained in the last several sections. For comparisons, Fig. 1(e) shows a result using anisotropic bilateral filter. It almost has no different with original image, this is because the original image contains most of low contrast region with almost uniform colors. Fig. 1(f) shows a result by shock filter. Compared with Fig. 1(d), this result contains hard, stiff and inflexible stream lines, while Fig. 1(f) 
contains soft stream flows pleasantly in visual sense.

More results are shown in Fig. 2, in which (a) is an original image and (b) is a result by adapting directly an anisotropic band-pass filter to the original image. The edges in image have been preserved and the regions with low contrast are smoothed. Only few of stream lines are created in the low contrast areas. Fig. 2(c) and (e) are trilateral filtered results with different standard deviation in image geometrical domain space. The standard deviation in image domain space for the results (c) and (e) are 0.5 and 1.5 respectively. A smaller standard deviation will create smaller blocky regions such as in Fig.2 (c), while a larger standard deviation will generate larger blocky region such as in Fig.2 (e). Moreover, standard deviation decides also the width of stream flows created by anisotropic band-pass filter. This is very obvious by observing Fig.2 (d) in which the stream flows are thinner and (f) in which the stream flows are thicker. The trilateral filter creates watercolor-like painting when using a suitable standard deviation. We found that it is better to let this standard deviation be under 2.0. If the standard deviation is too large, the image will be smoothed in a larger scale and almost no stream flows will be created, and the computation cost will be more expensive. Moreover, details in image will almost disappear. For all experimental results, the standard deviation in color range space is set to be about 31.6 empirically, and the standard deviation in gradient space is recommended to be in the range $[0.05,0.3]$. Another standard deviation for tensor smoothing controls the length of created soft stream flows, and we used $\rho=2 \sigma_{e}$ in all of experiments.

Figure 3 illustrates another example of our experiments. This image contains most of low contrast regions proportionally. The image is smoothed while stream flows are generated especially in low contrast regions. The detail textures with small scales such as in regions of ground and surfaces of chimneys are also smoothed. Most of edges and corners are well preserved and enhanced in a larger scale. It should be noted that some of important areas such as small windows become smaller in the chimneys since a relative larger standard deviation is employed.

More experimental result is shown in Fig.4 in which (a) is an original image with a little girl and flowers. This image contains large scale textures with less low contrast regions. Fig.4 (b) is the final filtered result image. The regions with low contrast are smoothed and the feature of edges and corners is well preserved and enhanced.

Our trilateral filter has different weighting functions compared with those proposed within the related work for different purposes and it is hard to compare the performance with them. Since the evaluation of the algorithm also becomes difficult, visual observation seems a probable way to evaluate the quality. Our algorithm suffers time-consuming problem similar to bilateral filter. We mainly emphasized on the development of algorithm, the code is not optimized which runs on a notebook with CPU $1.8 \mathrm{GHz}$. Time complexities heavily depends on image sizes and parameters. Fig. 1 and 2 with size 512 × 341 cost about 15 seconds.

The evaluation of NPR images are very difficult. Objective measurements for quality are hard to design [36], while subjective measurements depending on individualities who have different culture backgrounds. However, by visually observing our results and compared with the original images, the stream flows are well generated and the appearances are charming and attractive.

\section{Conclusion}

We proposed a new approach for creating artistic painting with soft stream flows by image filtering method. The algorithm is completely automatic by given several parameters. The filter in the algorithm consists of two components: a trilateral filter and an anisotropic band-pass filter. The trilateral filter draws blocky regions in the direction perpendicular to the edge, the anisotropic band-pass filter average image regions in an ellipse for creating soft stream flows while preserving and enhancing edges and corners. The proposed approach can be applied to create artistic painting-like images and produce soft stream flows with pleasant and harmonic visual effects. For our future work, we are now making efforts to find solutions for GPU-based implementation to accelerate our algorithm. We also are investigating how to use computational intelligence techniques such as machine learning for designing filter coefficients for artistic image painting.

\section{Acknowledgements}

The authors would like to thank Mr. Kurabar who kindly allowed us to use his original photo named "The three primary colors" in Fig. 1(a). The photo is allocated at http://maruta. be/alpha/170. This work is a part of researches supported by Chunhui project of the Education Ministry of China under Grant No. Z2012100 and No. Z2014020 and National Nature Science Foundation of China under Grant No.61462072.

\section{References}

[1] B. Gooch and A. Gooch, Non-Photorealistic Rendering, A K Peters, Ltd., 2001.

[2] T. Strothotte and S. Stefan, Non-Photorealistic Computer Graphics: Modelling, Rendering and Animation, Morgan Kaufmann, 2002.

[3] O. Deussen, S. Hiller C. Van Overveld and T. Strothotte, Floating Points: A Method for Computing Stipple Drawings, Computer Graphics Forum, Vol.19, No.3, 40-51, 2000.

[4] A. Secord, Weighted Voronoi Stippling, In Proc NPAR, ACM Press, New York, pp.37-43, 2002.

[5] Kim, D., Son, M., Lee, Y., Kang, H., and Lee, S. Feature-guided Image Stippling. In Proceedings of Comput. Graph. Forum. 2008, pp. 1209-1216.

[6] M. P. Salisbury, S. E. Anderson, R. Barzel, and D. H. Salesin, Interactive Pen-and-Ink Illustration. In ACM SIGGRAPH 94 Conference Proceedings, pp. 101-108, July 1994. 
[7] D. Decarlo, A. Finkelstein, S. Rusinkiewicz and A. Santella, Suggestive Contour for Conveying Shape, In Proceedings of ACM SIGGRAPH, pp. 848-855, 2003.

[8] T. Judd, F.Durand and E. Andelson, Apparent Ridges for Line Drawing, In Proceeding of ACM SIGGRAPH, 2007.

[9] H. Kang, S. Lee, C. Chui. "Coherent Line Drawing". Proc. ACM Symposium on Non-photorealistic Animation and Rendering, pp. 43-50, San Diego, CA, 2007.

[10] M. Son, H. Kang, Y. Lee, S. Lee. "Abstract Line Drawings from 2D Images". Proc. Pacific Graphics, IEEE Press, pp. 333-342, Maui, Hawaii, 2007.

[11] A. Hertzmann, Tutorial: A Survey of Stroke-Based Rendering, IEEE Computer Graphics and Application, Vol.23, No.4, pp. 70-81, 2003.

[12] A. Hertzmann, Painterly Rendering with Curved Brush Strokes of Multiple Sizes, In Proceeding of the 25th Annual Conference on Computer Graphics and Interactive Techniques (SIGGRAPH 1998), pp. 453-460.

[13] K. Zeng, M. Zhao, C. Xiong, and S.C. Zhu, "From image parsing to painterly rendering", presented at ACM Trans. Graph., 2009.

[14] G. Papari, N. Petkov, and P. Campisi, Artistic Edge and Corner Enhancing Smoothing, IEEE Trans. Image Processing, Vol. 16, No. 10, pp.2449-2462, 2007.

[15] H. Kang, S. Lee, and C. K. Chui, Flow-Based Image Abstraction, IEEE Trans. Visualization and Computer Graphics, Vol. 15, No. 1, pp. 62-76, 2009.

[16] J. E. Kyprianidis and H. Kang, Image and Video Abstraction by Coherence-Enhancing Filtering, Computer Graphics Forum, Vol. 30, No. 2, (Proceedings Eurographics 2011)

[17] M. Nixon, and A. Aguado, Feature Extraction and Image Processing (2nd. ed.), Elsevier Ltd. 2008.

[18] C. Tomasi, and R. Manduchi, Bilateral Filtering for Gray and Color Images, In Proc. of IEEE International Conference on Computer Vision, pp.839-846, 1998.

[19] K. He, J. Sun and X. Tang, Guided Image Filter, IEEE Trans. On Pattern Analysis and Machine Intelligence, Vol. 35, No. 6, pp.1397-1409, 2013.

[20] M. Kuwahara, K. Hachimura, S. Ehiu, and M. Kinoshita, Processing of ri-angiocardiographic images, In Digital Processing of Biomedical Images, New York: Plenum, pp. 187-203, 1976.

[21] J. E. Kyprianidis, H. Kang, and J. Dollner, Image and Video Abstraction by Anisotropic Kuwahara Filtering, Computer Graphics Forum, Vol. 28, No.7, pp.1955-1963, 2009.

[22] M. Kass and D. Pesare, Coherent Noise for Non-Photorealistic Rendering, ACM Trans. On Graphics, Vol. 30, No. 4, Article 30, July 2011 .
[23] M. Zhao and S. Zhu, Abstract Painting with Interactive Control of Perceptual Entropy, ACM Trans. On Applied Perception, Vol. 10, No. 1, February 2013.

[24] N. Xie, H. Hachiya and M. Sugiyama, Artist Agent: A Reinforcement Learning Approach to Automatic Stroke Generation in Oriental Ink Painting, Proc. Of the $29^{\text {th }}$ International Conference on Machine Learning, pp.153-160, 2012.

[25] N. Xie, H. Hachiya and M. Sugiyama, Artist Agent: A Reinforcement Learning Approach to Automatic Stroke Generation in Oriental Ink Painting, IEICE Trans. INF. \& SYST. Vol. E96-D, No. 5, May 2013.

[26] S. Osher and L. I. Rudin, Feature-Oriented Image Enhancement Using Shock Filter, SIAM Journal on Numerical Analysis, Vol. 27, 919-940, 1990.

[27] J. Werckert, Coherence-Enhancing Diffusion of Color Image, Image and Vision Computing, Vol.17, No. 3, pp. 201-212, 1999.

[28] J. Werckert, Coherence-Enhancing Shock Filters, Pattern Recognition, Lect. Notes in Comput. Sc. 2781, pp. 1-8, 2003.

[29] J. E. Kyprianidis, and J. Dollner, Image Abstraction by Structure Adaptive Filtering, In Proc. EG UK Theory and Practice of Computer Graphics, pp. 51-58, 2008.

[30] H. Kang and S. Lee, Shape-simplifying Image Abstraction, Computer Graphics Forum, Vol. 27, No. 7, pp. 1773-1780, 2008.

[31] M. Bertalmio, G. Sapiro, V. Caselles and C. Ballester, Image Inpainting, Proceedings of SIGGRAPH 2000, New Orleans, USA, July 2000.

[32] K. Inoue and K. Urahama, Anisotropic Bilateral Filters for Edge-Preserving Stripe Enhancement, ITE, Vol. 58, No. 1, pp.115-120, 2004.

[33] P. Choudhury and Jack Tumblin, The Trilateral for High Contrast Images and Meshes, Erographics Symposium on Rendering 2003, pp.186-196.

[34] J. B. Shen, S. F. Fang, H. L. Zhao and X. G. Jin, Fast Approximation of Trilateral Filter for Tone Mapping Using a Signal Processing Approach, Signal Processing, Vol. 89, No. 5, May 2009, pp. 901-907.

[35] W. C. K. Wong, A. C. S. Chung and S. C. H. Yu, Trilateral Filtering for Biomedical Images, in Proc. IEEE Int. Symp. Biomedical Imaging, 2004, pp.820-823.

[36] D. Mould, Authorial Subjective Evaluation of Non-Photorealistic Images, NPAR '14 Proceedings of the Workshop on Non-Photorealistic Animation and Rendering, Pages 49-56. 